\title{
LONG-TERM COEXISTENCE FOR A COMPETITIVE SYSTEM OF SPATIALLY VARYING GRADIENT REACTION-DIFFUSION EQUATIONS
}

\author{
ANDREI KOROBEINIKOV, JOHN NORBURY AND GRAEME C. WAKE
}

MACSI, Department of Mathematics and Statistics, University of

Limerick, Ireland

andrei.korobeinikov@ul.ie

Mathematical Institute, University of Oxford, 24-29 St Giles, Oxford OX1 3LB, UK

norbury@maths.ox.ac.uk

Institute of Information and Mathematical Sciences, Massey

University at Albany, Auckland, New Zealand

g.c.wake@massey.ac.nz

\author{
AMS Classification \\ 92D30 (primary), 34D20 (secondary) \\ Proposed Running Head \\ Competing reaction-diffusion equations
}


Abstract. Spatial distribution of interacting chemical or biological species is usually described by a system of reaction-diffusion equations. In this work we consider a system of two reactiondiffusion equations with spatially varying diffusion coefficients which are different for different species and with forcing terms which are the gradient of a spatially varying potential. Such a system describes two competing biological species. We are interested in the possibility of long-term coexistence of the species in a bounded domain. Such long-term coexistence may be associated either with a periodic in time solution (usually associated with a Hopf bifurcation), or with time-independent solutions. We prove that no periodic solution exists for the system. We also consider some steady-states (the time-independent solutions) and examine their stability and bifurcations.

Key words: Reaction-diffusion equations, existence and multiplicity of steady-states, periodic solution, Lyapunov stability, bifurcations

Acknowledgement. We thank the referees for there comments and the suggestion that led to the conjecture in Remark 2.4.

AK acknowledges support of Japan Society for the Promotion of Science, through Project 17540099, and of the Science Foundation Ireland Mathematics Initiative though MACSI.

\section{INTRODUCTION}

A model describing interaction of two competing biological species with a possibility of non-homogeneous spatial distribution usually consists of a coupled set of reaction-diffusion equations with logistic population growth and some form of competition (see e.g. Okubo et al. (1989); Murray (1989)). Based on an idea that "the interaction is 
(to a degree) unknown in detail", Norbury and Wake (1993) incorporated a quadratic dependence for the interaction logistic terms into a mathematical model. To describe the spatial spread of two competing species - US original grey and UK indigenous red squirrels in the UK - they suggested a system of two reaction-diffusion equations

$$
u_{t}-u_{x x}=\lambda u\left(1-u^{2}-a^{2} v^{2}\right), \quad v_{t}-v_{x x}=\lambda v\left(b^{2}-a^{2} u^{2}-c^{2} v^{2}\right)
$$

where $u(x, t)$ and $v(x, t)$ are densities of the two species, $a, b, c$ are positive constants and $\lambda$ is a positive parameter; of course, only the case $u, v \geq 0$ is feasible for a population or concentration model. However in some cases both positive and negative solutions can be made physically relevant after scaling of the form $u=\alpha+\beta \widetilde{u}, v=\gamma+\delta \widetilde{v}$ (since $u_{x}=v_{x}=$ 0 translates to $\widetilde{u}_{x}=\widetilde{v}_{x}=0$, and $u_{t}=\beta \widetilde{u}_{t}$, etc.) which transforms the reaction term without changing the rest of the equations. Depending on the nature of an application (or the boundary conditions), we may wish to consider either strictly positive (non-negative) solutions, or solutions of both signs.

A remarkable feature of this model is that the system (1.1) is a gradient system: the right parts of the equations (1.1) are partial derivatives, $G_{u}=\lambda u\left(1-u^{2}-a^{2} v^{2}\right)$ and $G_{v}=\lambda v\left(b^{2}-a^{2} u^{2}-c^{2} v^{2}\right)$ respectively, of the potential

$$
G(u, v)=\frac{\lambda}{2}\left(u^{2}-\frac{1}{2} u^{4}-a^{2} u^{2} v^{2}+b^{2} v^{2}-\frac{1}{2} c^{2} v^{4}\right)
$$

Further, there is a symmetry, or a group action $u \rightarrow-u$ and $v \rightarrow-v$ under which the potential (and hence the system) is invariant. The model may be generalised to the multidimensional case in a straightforward way. 
In modelling situations gradient reaction-diffusion systems can and do arise from physically- or physiologically-derived situations when there is some type of "overall global conservation" occurring. In population biology, from which this model arose, this can occur when species are competing with each other for resources. The model in equation (1.1) was determined both by looking at the data and by consideration of the specific growth rate of each component species in the presence of the other (Okubo et al., 1989). Subsequent scaling of the variables enabled it to be cast in the form of a gradient system. Care must be taken in doing this to ensure that the boundary conditions are not inadvertently altered so that the system no longer satisfies the conditions of the results in Section 2. In the specific case of the competing squirrel populations this did not occur as it involved just a simple scaling of the populations. That is, it is a "robust gradient system".

Norbury and Wake (1993) focused on the possibility of long-term coexistence of two competing species, that is possibility of static (timeindependent) or periodic solutions. A time-independent spatial distribution of the densities of the two species $u(x)$ and $v(x)$ satisfies the equations

$$
\frac{d^{2} u}{d x^{2}}=-\lambda u\left(1-u^{2}-a^{2} v^{2}\right), \quad \frac{d^{2} v}{d x^{2}}=-\lambda v\left(b^{2}-a^{2} u^{2}-c^{2} v^{2}\right)
$$

defined on an open interval $0<x<1$ (the actual length of the domain was scaled into $\lambda$ ). To close the system (1.2), boundary conditions should be added. The no-flux boundary conditions

$$
u_{x}(0)=v_{x}(0)=0, \quad u_{x}(1)=v_{x}(1)=0
$$


reflect a situation of impermeable boundaries. Under a "hostile environment" assumption the Dirichlet boundary conditions

$$
u(0)=v(0)=0, \quad u(1)=v(1)=0
$$

can be used. The latter case was considered by Norbury and Wake (1993).

The system (1.2) is a Hamiltonian system with independent variable $x$ : the equations (1.2) are the Euler-Lagrange equations for the functional

$$
I=\int_{0}^{1}\left(\frac{1}{2}\left(u_{x}^{2}+v_{x}^{2}\right)-G(u, v)\right) d x
$$

The function

$$
E(x)=\frac{1}{2}\left(u_{x}^{2}+v_{x}^{2}\right)+G(u, v)
$$

is the first integral of the system (1.2): that is for any solution of the system (1.2) the function $E(x)$ is constant for all $x$. In particular in the case of one spatial dimension for the homogeneous Neumann boundary conditions $E(x)=G(u(0), v(0))=G(u(1), v(1))$ for all $x \in[0,1]$.

In this work we consider existence of long term co-existing solutions - both periodic in time and steady-state coexistence - of two species in a multi-dimensional bounded simply connected region $\Omega \subset$ $\mathbf{R}^{n}$. We also extend equation (1.1) so that different species diffusions and more general interaction potential functions $W(\mathbf{x}, u, v)$ are allowed. In Section 2 we consider the possibility of periodicity in time for this system, and show that no periodicity is possible. We then go on in Sections 3 and 4 to consider steady-states and to classify them as regards to stability. In Sections 5, 6 and 7 we consider some explicit 
solution which may exist for biological system with competition, and their bifurcations.

\section{Nonexistence of PERIOdic SOlutions}

Speed of spatial propagation differs for different biological species. Consequently the corresponding diffusion coefficients which characterise the speed of spatial propagation are also different. Chemical species also have different diffusion rates. Further, the rate of spatial propagation (and hence the corresponding diffusion coefficient) may vary in space depending, for example, on environmental conditions. In chemistry it is also possible to have diffusion rates depending on the location in a domain: for instance, varied in space background temperature can lead to varying in space diffusion rates.

Furthermore, carrying capacities, reproduction rates and competition rates may also vary in space, depending on varying natural conditions. That is the potential and reaction terms can depend on location in space as well.

We will consider two competing species in a multi-dimensional region $\Omega \subset \mathbf{R}^{n}$. Of course $n=2$ for biological species (or $n=3$ for some bacteria or more generally in chemical reactions); however, the results of this paper are valid for any number of dimensions.

We assume that two interacting species with densities $u(\mathbf{x}, t)$ and $v(\mathbf{x}, t)$ have different and varying in space diffusion rates, denoting them $D_{1}(\mathbf{x})$ and $D_{2}(\mathbf{x})$ respectively, and that the potential $W(\mathbf{x}, u, v)$ can vary in space as well. The densities of the species satisfy the 
equations

$$
\begin{aligned}
& \frac{\partial u(\mathbf{x}, t)}{\partial t}=\nabla \cdot\left(D_{1}(\mathbf{x}) \nabla u(\mathbf{x}, t)\right)+\frac{\partial W(\mathbf{x}, u, v)}{\partial u} \\
& \frac{\partial v(\mathbf{x}, t)}{\partial t}=\nabla \cdot\left(D_{2}(\mathbf{x}) \nabla v(\mathbf{x}, t)\right)+\frac{\partial W(\mathbf{x}, u, v)}{\partial v}
\end{aligned}
$$

defined on a bounded simply connected region $\mathbf{x} \in \Omega \subset \mathbf{R}^{n}$ for $t>0$, with boundary and initial conditions

$$
\begin{aligned}
& D_{1}(\mathbf{x}) \frac{\partial u}{\partial n}+B(\mathbf{x}) \cdot\left(u-u_{a}(\mathbf{x})\right)=0 \quad \text { and } \\
& D_{2}(\mathbf{x}) \frac{\partial v}{\partial n}+C(\mathbf{x}) \cdot\left(v-v_{a}(\mathbf{x})\right)=0 \quad \text { for } \quad \mathbf{x} \in \partial \Omega, \quad t \geq 0 \\
& u(\mathbf{x}, 0)=\phi(\mathbf{x}), \quad v(\mathbf{x}, 0)=\psi(\mathbf{x}) \quad \text { for } \quad \mathbf{x} \in \Omega
\end{aligned}
$$

Here $B(\mathbf{x}), C(\mathbf{x}), u_{a}(\mathbf{x})$ and $v_{a}(\mathbf{x})$ are time-independent functions, defined on the smooth boundary $\partial \Omega$. The diffusion rates $D_{1}(\mathbf{x})$ and $D_{2}(\mathbf{x})$ are continuously differentiable and strictly positive on the closed domain $\bar{\Omega}$; the potential $W(\mathbf{x}, u, v) \in C^{2}$.

The following Theorem generalises a result of Norbury and Wake (1993) on nonexistence of periodic solution to the multidimensional case with non-homogeneous boundary conditions (2.2) and with different varying in space diffusion coefficients.

Theorem 2.1. There is no non-constant periodic in time $t$ solution for the initial-boundary value problem (2.1)-(2.2).

Proof. Let us consider the functional (which is a generalisation of that in $(1.5))$

$$
\begin{array}{cc}
F(t)= & \int_{\Omega}\left(\frac{1}{2}\left(D_{1}(\mathbf{x})(\nabla u)^{2}+D_{2}(\mathbf{x})(\nabla v)^{2}\right)-W(\mathbf{x}, u, v)\right) d V \\
& +\frac{1}{2} \oint_{\partial \Omega}\left(B(\mathbf{x})\left(u-u_{a}(\mathbf{x})\right)^{2}+C(\mathbf{x})\left(v-v_{a}(\mathbf{x})\right)^{2}\right) d S \\
7
\end{array}
$$


If there is a periodic solution $u(t, x), v(t, x)$, then $F(t)$ is periodic as well. Nevertheless, noting that by the Gauss divergence theorem

$$
\int_{\Omega} \nabla \cdot\left(u_{t} D \nabla u\right) d V=\oint_{\partial \Omega} u_{t} D \nabla u \cdot \mathbf{n} \cdot d S=\oint_{\partial \Omega} u_{t} D \frac{\partial u}{\partial n} d S,
$$

we find

$$
\begin{aligned}
\frac{d F(t)}{d t}= & \int_{\Omega}\left(D_{1} \nabla u \cdot \nabla u_{t}+D_{2} \nabla v \cdot \nabla v_{t}-W_{u} \cdot u_{t}-W_{v} \cdot v_{t}\right) d V \\
& +\oint_{\partial \Omega} B\left(u-u_{a}\right) u_{t} d S+\oint_{\partial \Omega} C\left(v-v_{a}\right) v_{t} d S \\
= & \int_{\Omega}\left(\nabla \cdot\left(u_{t} D_{1} \nabla u\right)-u_{t} \nabla\left(D_{1} \nabla u\right)-W_{u} \cdot u_{t}\right) d V \\
& +\int_{\Omega}\left(\nabla \cdot\left(v_{t} D_{2} \nabla v\right)-v_{t} \nabla\left(D_{2} \nabla v\right)-W_{v} \cdot v_{t}\right) d V \\
& +\oint_{\partial \Omega} B\left(u-u_{a}\right) u_{t} d S+\oint_{\partial \Omega} C\left(v-v_{a}\right) v_{t} d S \\
= & -\int_{\Omega} u_{t}\left(\nabla\left(D_{1} \nabla u\right)+W_{u}\right) d V+\oint_{\partial \Omega}\left(D_{1} \frac{\partial u}{\partial n}+B\left(u-u_{a}\right)\right) u_{t} d S \\
& -\int_{\Omega} v_{t}\left(\nabla\left(D_{2} \nabla v\right)+W_{v}\right) d V+\oint_{\partial \Omega}\left(D_{2} \frac{\partial v}{\partial n}+C\left(v-v_{a}\right)\right) v_{t} d S \\
= & -\int_{\Omega}\left(u_{t}^{2}+v_{t}^{2}\right) d V \leq 0,
\end{aligned}
$$

and $\frac{d F(t)}{d t}=0$ only for time-independent solutions. This contradicts the assumption about the periodicity of the functional. Hence there are no periodic solutions for the system, in view of the monotonicity of $F(t)$.

The theorem holds for $B(\mathbf{x}), C(\mathbf{x}), u_{a}(\mathbf{x})$ and $v_{a}(\mathbf{x})$ of both signs, however we require physically realistic boundary conditions below.

The following Corollary is obvious.

Corollary 2.2. No Hopf bifurcation is possible for the initial-boundary value problem (2.1)-(2.3). 
Remark 2.3. Theorem 2.1 and Corollary 1 hold for gradient systems of all sizes, and a single reaction-diffusion equation, whose forcing term always may be considered as a gradient of a potential, provides a particularly important case.

Remark 2.4. Gradient reaction-diffusion systems therefore have no persistent periodic solutions, and so limit cycles cannot be an attractor for the transient solutions. If the solutions exist for arbitrarily long time (as they certainly do for most modelling situations), there will be steady-state attractors (excepting chaotic solutions of course). The question then arises as to whether reaction-diffusion systems which are almost in gradient form, that is,

$$
\mathbf{f}(\mathbf{x}, u, v)=\nabla W+\epsilon \mathbf{g}(\mathbf{x}, u, v),
$$

where $\mathbf{g}$ is a non-gradient vector function and $\epsilon<<1$, give rise to periodic solutions under some conditions? This is an open question. We conjecture that if they do, then these solutions are singular in the limit of $\epsilon \rightarrow 0$. The nature of their disappearance needs to be considered. If they are, as we expect, of order $O\left(\epsilon^{-1}\right)$, as $\epsilon \rightarrow 0$, then this will be highly relevant in the modelling situation as they are most likely to be infeasible solutions for sufficiently small $\epsilon$.

We now proceed to analyse the existence of steady-state solutions of the problem (2.1)-(2.3). 


\section{Steady-State Solutions}

Steady-state solutions of the system (2.1)-(2.3), that is time-independent distributions of the densities $u(\mathbf{x}), v(\mathbf{x})$ satisfy the equations

$$
\begin{aligned}
& \nabla 3: 1\left(D_{1} \nabla u\right)=-\frac{\partial W}{\partial u}, \quad \nabla \cdot\left(D_{2} \nabla v\right)=-\frac{\partial W}{\partial v}, \quad \text { for } \quad \mathbf{x} \in \Omega \subset \mathbf{R}^{n}, \\
& D_{a} \cdot \frac{\partial u}{\partial n}=-B\left(u-u_{a}\right), \quad D_{2} \frac{\partial v}{\partial n}=-C\left(v-v_{a}\right), \quad \text { for } \quad \mathbf{x} \in \partial \Omega .
\end{aligned}
$$

Here $D_{1}(\mathbf{x}), D_{2}(\mathbf{x})>0$ are diffusion rates, $W(\mathbf{x}, u, v)$ is a potential, and $B(\mathbf{x}), C(\mathbf{x}), u_{a}(\mathbf{x})$ and $v_{a}(\mathbf{x})$ are given functions. We assume that further $B(\mathbf{x}), C(\mathbf{x}) \geq 0$, since the case $B(\mathbf{x}), C(\mathbf{x})<0$ is usually not of practical interest.

The following result is not unexpected, however we include it here for completeness. In fact, it is another corollary of Theorem 2.1.

Theorem 3.1. If the potential $W(\mathbf{x}, u, v)$ is bounded from above and $B(\mathbf{x}), C(\mathbf{x}) \geq 0$, then, as $t \rightarrow \infty$, a solution of the initial-boundary value problem (2.1)-(2.3) tends to a solution of the time-independent boundary value problem (3.1), (3.2).

In other words, there is an $\omega$ limit set of the initial-boundary value problem (2.1)-(2.3), and this $\omega$ limit set is composed of time-independent solutions.

Proof. The potential $W(\mathbf{x}, u, v)$ is bounded from above, that is

$$
W(\mathbf{x}, u, v) \leq \bar{W}=\max _{\mathbf{x}, u, v} W
$$


and $B(\mathbf{x}), C(\mathbf{x}) \geq 0$. Hence the functional (2.4) is bounded from below,

$$
\begin{aligned}
F(t)= & \int_{\Omega}\left(\frac{1}{2}\left(D_{1}(\mathbf{x})(\nabla u)^{2}+D_{2}(\mathbf{x})(\nabla v)^{2}\right)-W(\mathbf{x}, u, v)\right) d V \\
& +\frac{1}{2} \oint_{\partial \Omega}\left(B(\mathbf{x})\left(u-u_{a}(\mathbf{x})\right)^{2}+C(\mathbf{x})\left(v-v_{a}(\mathbf{x})\right)^{2}\right) d S \\
\geq & \int_{\Omega}\left(\frac{1}{2}\left(D_{1}(\mathbf{x})(\nabla u)^{2}+D_{2}(\mathbf{x})(\nabla v)^{2}\right)-W(\mathbf{x}, u, v)\right) d V \\
\geq & -\int_{\Omega} W(\mathbf{x}, u, v) d V \geq-\bar{W} \int_{\Omega} d V=-\bar{W}\|\Omega\|,
\end{aligned}
$$

and therefore may be considered as a Lyapunov function for the system (2.1)-(2.3) (Barbashin, 1970; Lyapunov, 1992; La Salle and Lefschetz, 1961). By the proof of Theorem 2.1, $\frac{d F}{d t}=-\int_{\Omega}\left(u_{t}^{2}+v_{t}^{2}\right) d V \leq 0$, that is the functional monotonically decreases with the time $t$ for all time-dependent solutions of the system (2.1)-(2.3). Since the functional $F(t)$ is bounded from below, there is a finite limit, $\bar{F} \geq-\bar{W}\|\Omega\|$, such that $\lim _{t \rightarrow \infty} F(t)=\bar{F}$. Since $\frac{d F}{d t}=0$ only when $u_{t}=v_{t}=0$, this limit is achieved on a pair of time-independent functions $(u(\mathbf{x}), v(\mathbf{x}))$ which forms a time-independent solution. Since the system (3.1), (3.2) is the Euler-Lagrange equation for the functional $F$, this pair of functions $u(\mathbf{x}), v(\mathbf{x})$ satisfies both problems, (2.1)-(2.2) and (3.1), (3.2).

Corollary 3.2. A time-independent solution of the system (2.1)-(2.2) is stable iff it minimises the functional (2.4) on a class of functions satisfying the equations and boundary conditions. A solution is asymptotically stable iff the corresponding minimum of the functional is isolated.

Proof. By the Lyapunov asymptotic stability theorem (Barbashin, 1970; Lyapunov, 1992; La Salle and Lefschetz, 1961) with the functional (2.4) as a Lyapunov function. 


\section{Spatially uniform Steady-States}

If the boundaries of a region are given by lakes or rivers, then for the biological species problem no flux, or homogeneous Neumann boundary conditions,

$$
u_{n}(\mathbf{x})=v_{n}(\mathbf{x})=0 \quad \text { for } \quad \mathbf{x} \in \partial \Omega,
$$

may be applied naturally. In the case of the homogeneous Neumann boundary value problem a very specific steady-state solution, a spatially uniform steady-state which does not depend on the spatial variable $\mathbf{x}$, can exist. Spatially uniform functions $u, v$ satisfy the homogeneous Neumann boundary conditions naturally. For a spatially uniform steady-state $\nabla u=\nabla v=0$ holds throughout $\Omega$, and hence $u, v$ satisfy the equations

$$
W_{u}(\mathbf{x}, u, v)=0, \quad W_{v}(\mathbf{x}, u, v)=0 \quad \text { for all } \quad \mathbf{x} \in \Omega .
$$

That is the spatially uniform steady-states correspond to critical points of the potential function $W(\mathbf{x}, u, v)$ under the condition that these critical points do not depend on the spatial variable $\mathbf{x}$. Of course, such a solution can exist only if the location of these critical points in the $u, v$ space does not depend on the spatial variable $\mathbf{x}$. For example, such a situation is possible if the potential function $W(\mathbf{x}, u, v)$ does not depend on $\mathbf{x}$. However, spatial independence of the potential $W(\mathbf{x}, u, v)$ is not a necessary condition for existence of spatially uniform steady-states; for example for a potential of the form $W(\mathbf{x}, u, v)=g(\mathbf{x}) \cdot \widetilde{W}(u, v)$, where $\widetilde{W}(u, v)$ does not depend on $\mathbf{x}$, existence of spatially uniform steady-states depends on whether or not the function $\widetilde{W}(u, v)$ has critical points. 
Note that dependence of the diffusion coefficients $D_{1}(\mathbf{x})$ and $D_{2}(\mathbf{x})$ on the spatial variable $\mathbf{x}$ does not affect the existence of the spatially uniform steady-states.

If the spatially uniform steady-states exist, then the following theorem shows their importance.

Theorem 4.1. If a homogeneous Neumann initial-boundary value problem has spatially uniform steady-states, then those which correspond to maxima of the potential $W(\mathbf{x}, u, v)$ are asymptotically stable, while those which correspond to saddle points or minima are unstable. If a maximum is unique then the corresponding spatially uniform steadystate is globally asymptotically stable.

Proof. By Corollary 3.2, a steady-state of the system (2.1)-(2.2) is stable if it minimises on the class of solutions of the system the functional (2.4), which in the case of the homogeneous Neumann boundary conditions takes the form

$$
F(t)=\int_{\Omega}\left(\frac{1}{2}\left(D_{1}(\mathbf{x})(\nabla u)^{2}+D_{2}(\mathbf{x})(\nabla v)^{2}\right)-W(\mathbf{x}, u, v)\right) d V
$$

For a spatially uniform solution $\nabla u=\nabla v=0$ holds throughout $\Omega$, and the spatially uniform steady-states correspond to critical points of the potential $W(\mathbf{x}, u, v)$. Hence those spatially uniform steady-states which are isolated maxima (local ones, at least) of the potential $W(\mathbf{x}, u, v)$, minimise the functional (4.3) and are asymptotically stable (locally, at least) (Barbashin, 1970; Lyapunov, 1992; La Salle and Lefschetz, 1961).

It is remarkable that the spatially uniform solutions of the system provide not a relative (on a class of solutions of the problem) but an 
absolute (on $C^{2}$ ) minimum of the functional. Of course this result critically depends on the Neumann boundary conditions.

In the particular case of two competing biological species the potential

$$
G(u, v)=\frac{\lambda}{2}\left(u^{2}-\frac{1}{2} u^{4}-a^{2} u^{2} v^{2}+b^{2} v^{2}-\frac{1}{2} c^{2} v^{4}\right)
$$

is now considered as a generic example. In this case spatially uniform steady-states satisfy the equations

$$
G_{u}=\lambda u\left(1-u^{2}-a^{2} v^{2}\right)=0, \quad G_{v}=\lambda v\left(b^{2}-a^{2} u^{2}-c^{2} v^{2}\right)=0 .
$$

That is the spatially uniform steady-states are the points of intersection of the ellipses, $u^{2}+a^{2} v^{2}=1$ and $a^{2} u^{2}+c^{2} v^{2}=b^{2}$, and the axes $u=0$ and $v=0$ (see Fig. 4.1). Three solutions, $Q_{1}=(0,0), Q_{2}=(1,0)$ and $Q_{3}=(0, b / c)$, exist for all $a, b, c>0$; the fourth steady-state, the coexisting steady-state $Q_{4}=\left(\sqrt{\frac{c^{2}-a^{2} b^{2}}{c^{2}-a^{4}}}, \sqrt{\frac{b^{2}-a^{2}}{c^{2}-a^{4}}}\right)$, exists if either $c>a b$ and $b>a$, or $c<a b$ and $b<a$ hold simultaneously.

For all $\lambda, a, b, c>0$ the steady-state $Q_{1}=(0,0)$ is a local minimum of the function $G(u, v)$. Depending on the parameters values, four regular cases and one singular case are possible for the other steadystates (Fig. 4.1).

Case 1. If $c>a b$ and $b>a$ hold simultaneously, then the co-existing steady-state $Q_{4}$ exists and is the only maximum of the function $G(u, v)$ in the non-negative quadrant $u, v \geq 0$. The non-coexisting steadystates $Q_{2}=(1,0)$ and $Q_{3}=\left(0, \frac{b}{c}\right)$ are in this case saddle points.

Case 2. If $c<a b$ and $b<a$ hold simultaneously, then the co-existing steady-state $Q_{4}$ exists and is a saddle point of the function $G(u, v)$. The non-coexisting steady-states $Q_{2}$ and $Q_{3}$ are then local maxima. 


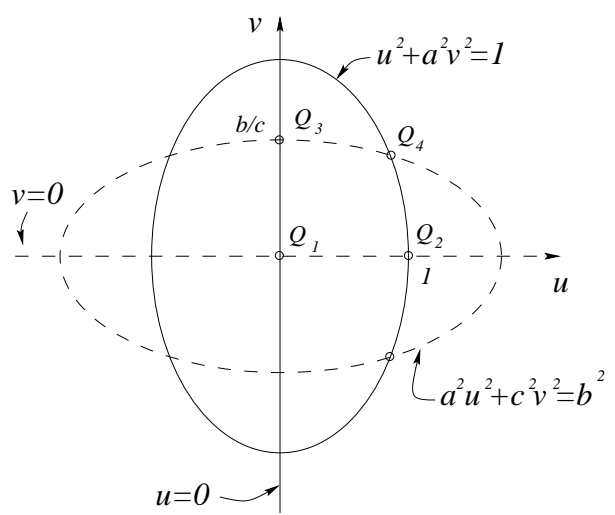

Case 1: $c>a b \& b>a$

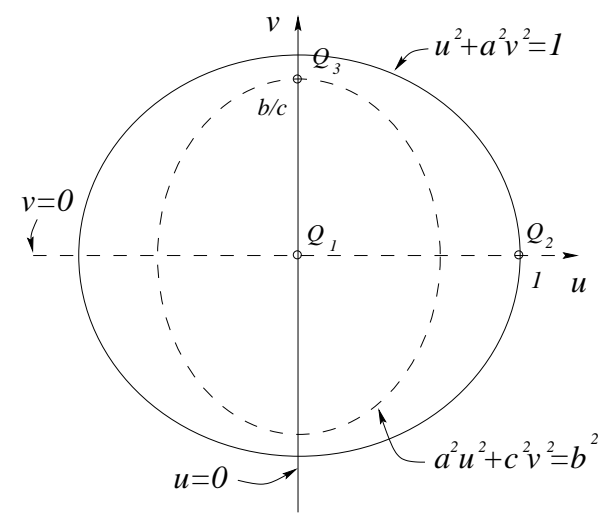

Case 3: $c>a b \& b<a$

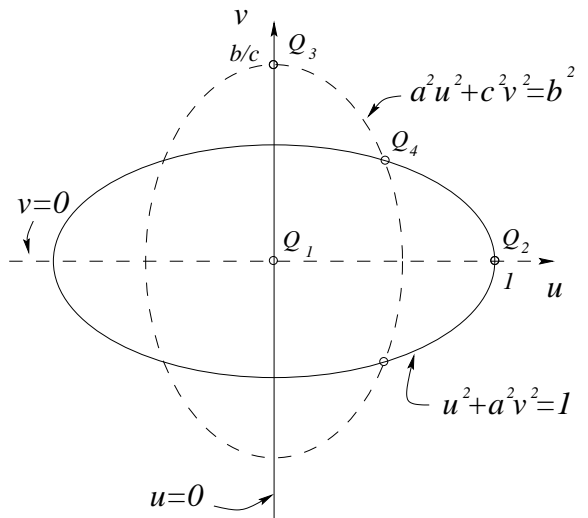

Case 2: $c<a b \& b<a$

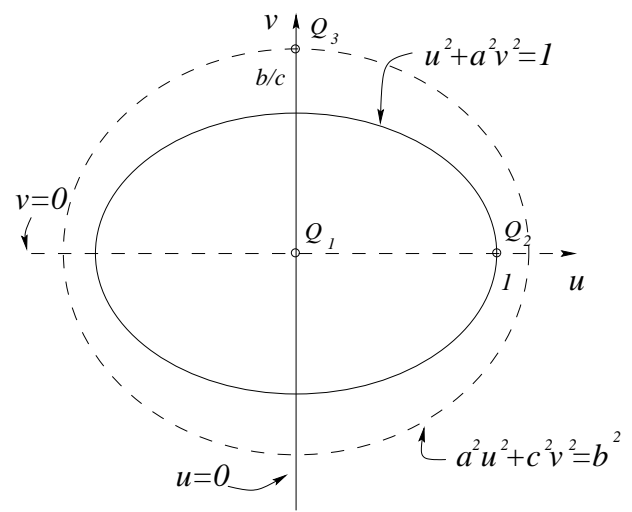

Case 4: $c<a b \& b>a$

Figure 4.1. Critical points of the potential function $G(u, v)=\frac{\lambda}{2}\left(u^{2}-\frac{1}{2} u^{4}-a^{2} u^{2} v^{2}+b^{2} v^{2}-\frac{1}{2} c^{2} v^{4}\right)$.

Case 3. If either $c \geq a b$ and $b<a$, or $c>a b$ and $b \leq a$, then the ellipses do not intersect, and the co-existing spatial uniform steadystate $Q_{4}$ does not exist. $Q_{2}$ is the unique maximum of the function $G(u, v)$, and $Q_{3}$ is a saddle point.

Case 4. The case when either $c \leq a b$ and $b>a$, or $c<a b$ and $b \geq a$ mirrors Case 3: now $Q_{3}$ is the unique maximum, while $Q_{2}$ is a saddle point. 
Case 5, when $c=a b$ and $b=a$ hold simultaneously, is a singular case. In this Case the ellipses coincide, and the whole ellipse $u^{2}+a^{2} v^{2}=1$ is the critical set of the function $W(u, v)$ and its maximum.

According to Theorem 4.1 the steady-state $Q_{1}=(0,0)$ is a minimum of the function $G(u, v)$ for all $\lambda, a, b, c>0$, and hence is unstable.

In the Case 1 the co-existing steady-state $Q_{4}$ is the only maximum of the function $G(u, v)$ in the non-negative quadrant $u, v \geq 0$ and is globally asymptotically stable. The non-coexisting steady-states $Q_{2}=$ $(1,0)$ and $Q_{3}=\left(0, \frac{b}{c}\right)$ are in this case saddle points, and hence they are unstable.

In the Case 2 the co-existing steady-state $Q_{4}$ is a saddle point of the function $G(u, v)$, and hence it is unstable. The non-coexisting steadystates $Q_{2}$ and $Q_{3}$ are local maxima of the function $G(u, v)$. They are asymptotically stable, and the set $\left\{Q_{2}, Q_{3}\right\}$ is the $\omega$ limit set of the system.

In the Case $3 Q_{2}$ is the unique maximum of the function $G(u, v)$ and is globally asymptotically stable. $Q_{3}$ is a saddle point and is unstable.

Case 4 mirrors Case 3: now $Q_{3}$ is the unique maximum and is globally asymptotically stable, while $Q_{2}$ is a saddle point and is unstable.

In the singular Case 5 each point of the ellipse $u^{2}+a^{2} v^{2}=1$ is stable, and the whole ellipse $u^{2}+a^{2} v^{2}=1$ is the $\omega$ limit set of the problem.

\section{Explicit solutions}

There are several cases when the system may be reduced to a single equation. If $u(\mathbf{x})$ and $v(\mathbf{x})$ are biological or chemical species concentrations, then a case when one of the species is absent is possible. If, say, $v(\mathbf{x}) \equiv 0$ then we consider a solution of the type $(u(\mathbf{x}), v(\mathbf{x}))=$ $\left(u^{*}(\mathbf{x}), 0\right)$. We henceforth refer to this as "non-coexisting" solutions. 
It is easy to see that such a non-coexisting solution exists if

$$
W_{v}\left(\mathbf{x}, u^{*}(\mathbf{x}), 0\right)=0
$$

for all feasible $u^{*}(\mathbf{x})$ and for all $\mathbf{x} \in \Omega$, and in this case the system reduces to the single equation

$$
\nabla\left(D_{1} \nabla u^{*}\right)=W_{u}\left(\mathbf{x}, u^{*}, 0\right)
$$

Likewise, assuming that $u(\mathbf{x}) \equiv 0$ we consider a solution of the type $(u(\mathbf{x}), v(\mathbf{x}))=\left(0, v^{*}(\mathbf{x})\right)$. If

$$
W_{u}\left(\mathbf{x}, 0, v^{*}(\mathbf{x})\right)=0
$$

for all feasible $v^{*}(\mathbf{x})$ and for all $\mathbf{x} \in \Omega$, the system (3.1), (3.2) reduces to the equation

$$
\nabla\left(D_{2} \nabla v^{*}\right)=W_{v}\left(\mathbf{x}, 0, v^{*}\right)
$$

Another case when the system (3.1), (3.2) may be reduced to a single equation is a "proportionate" solution such that

$$
\frac{v(\mathbf{x})}{u(\mathbf{x})}=k=\mathrm{const} \quad \text { for all } \quad \mathbf{x} \in \Omega,
$$

which may exist in some cases for the homogeneous Dirichlet or Neumann boundary conditions. As in the cases of the "non-coexisting" solution, the assumption (5.5) enables us to reduce the system (3.1) to a single differential equation for one undefined function, say

$$
\nabla\left(D_{1} \nabla u\right)=W_{u}(\mathbf{x}, u, k u)
$$


which is obtained by substituting (5.5) into (3.1). The above-mentioned non-coexisting solutions $(u(\mathbf{x}), v(\mathbf{x}))=\left(u^{*}(\mathbf{x}), 0\right)$ and $(u(\mathbf{x}), v(\mathbf{x}))=$ $\left(0, v^{*}(\mathbf{x})\right)$ are the particular cases of the type (5.5) solution, with $k=0$ for the former and $k=\infty$ for the latter.

This type of solution exists only if the constant parameter $k$, which satisfies the system (3.1), (3.2) and the identity (5.5), exists. This is possible, for instance, if the potential function $W(\mathbf{x}, u, v)$ and the diffusion coefficients $D_{1}(\mathbf{x})$ and $D_{2}(\mathbf{x})$ do not depend on $\mathbf{x}$. However, spatial independence of the potential $W(\mathbf{x}, u, v)$ and the diffusion coefficients $D_{1}(\mathbf{x})$ and $D_{2}(\mathbf{x})$ is neither a necessary, nor a sufficient condition for existence of the "proportional" solution: for example, for a potential of the form $W(\mathbf{x}, u, v)=g(\mathbf{x}) \widetilde{W}(u, v)$ and the diffusion coefficients of the form $D_{1}(\mathbf{x})=h(\mathbf{x}) \widetilde{D_{1}}$ and $D_{2}(\mathbf{x})=h(\mathbf{x}) \widetilde{D_{2}}$, where $\widetilde{W}(u, v)$ does not depend on $\mathbf{x}$, and $\widetilde{D_{1}}$ and $\widetilde{D_{2}}$ are constant, existence of this type of solution depends on the function $\widetilde{W}(u, v)$. In this case to satisfy the system (3.1), (3.2) and the identity (5.5) the undefined parameter $k$ must satisfy the equation

$$
k \widetilde{D}_{2} W_{u}(\mathbf{x}, u(\mathbf{x}), k u(\mathbf{x}))=\widetilde{D}_{1} W_{v}(\mathbf{x}, u(\mathbf{x}), k u(\mathbf{x})),
$$

which is obtained by substituting (5.5) into (3.1).

Remark 5.1. The "proportionate" solutions of the type (5.5) are not limited to the system $(3.1),(3.2)$, or a two-variable system only. It is easy to see that solutions of this type may exist for the homogeneous Dirichlet and Neumann boundary conditions for Hamiltonian and nonHamiltonian systems of $n$ equations for $n$ unknown functions $u_{i}, i=$ 
$1, \cdots, n$. Then $n-1$ conditions

$$
\frac{u_{i}}{u_{1}}=k_{i}
$$

for $n-1$ undefined constant parameters $k_{i}$ should be substituted into an original system to reduce the system of $n$ equations to the one differential equation for an unknown function $u_{1}$ and $n-1$ algebraic equations for the unknown parameters $k_{i}$. Of course, the possibility of a solution for the case $u_{1}(\mathbf{x}) \equiv 0$ must be considered as well.

6. The special Case of a homogeneous potential $G(u, v)$

We now consider a particular case of two competing biological species the system (3.1), (3.2) with the space-independent potential

$$
G(u, v)=\frac{\lambda}{2}\left(u^{2}-\frac{1}{2} u^{4}-a^{2} u^{2} v^{2}+b^{2} v^{2}-\frac{1}{2} c^{2} v^{4}\right)
$$

introduced in the Introduction, and constant and spatially independent diffusion coefficients $D_{1}$ and $D_{2}$. For the potential $G(u, v)$

$$
G_{u}(u, v)=\lambda u\left(1-a^{2} v^{2}-u^{2}\right), \quad G_{v}(u, v)=\lambda v\left(b^{2}-c^{2} v^{2}-a^{2} u^{2}\right),
$$

and hence the conditions (5.1) and (5.3) hold, which gives existence of non-coexisting solutions $(u(\mathbf{x}), v(\mathbf{x}))=\left(u^{*}(\mathbf{x}), 0\right)$ and $(u(\mathbf{x}), v(\mathbf{x}))=$ $\left(0, v^{*}(\mathbf{x})\right)$. Non-trivial solutions of the equations

$$
D_{1} \nabla^{2} u+\lambda u\left(1-u^{2}\right)=0
$$

and

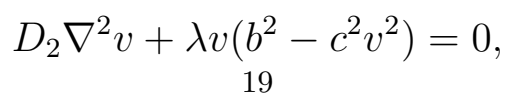


corresponding to the non-coexisting solutions, emanate from the trivial solution $u(\mathbf{x})=v(\mathbf{x}) \equiv 0$ at eigenvalues (see Fig. 6.1) of the linearised problem

$$
\begin{aligned}
& D_{i} \nabla^{2} \xi+\mu_{i} \xi=0 \quad \text { for } \quad \mathbf{x} \in \Omega \quad(i=1,2), \\
& B_{i}(\xi)=0 \text { for } \quad \mathbf{x} \in \partial \Omega .
\end{aligned}
$$

Here $\mu_{1}=G_{u u}(0,0)=\lambda$ and $\mu_{2}=G_{v v}(0,0)=\lambda b^{2}$.

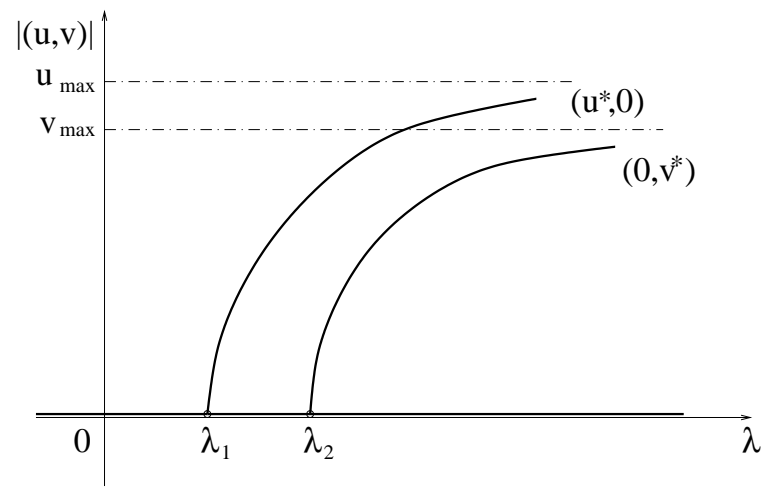

FiguRE 6.1. Bifurcation diagram for the non-coexisting solutions.

In the case of one space dimension the $n$th branch of these solutions, with eigenvalues $\mu_{i}^{n}=n^{2} \pi^{2} D_{i} / L$, where $L$ is the region size, exhibits $n-1$ nodal points where $u$ (or $v$, if the solution $\left(0, v^{*}(\mathbf{x})\right)$ is under consideration) equals zero (other than at the boundary). For $\lambda$ sufficiently large, $u$ (or $v$ ) approaches its maximum value almost everywhere.

We will further use the notation $u(\mathbf{x}, \lambda), v(\mathbf{x}, \lambda)$ to stress the dependence of the solution on these branches on the value of $\lambda$. We will focus on the first branch emanating from the first (the smallest) eigenvalue in view of the fact that other branches are just obtained from repeats of each of $u^{*}(\mathbf{x}, \lambda)$ and $v^{*}(\mathbf{x}, \lambda)$ with alternating signs. We also note that, in terms of looking at the corresponding time-dependent system, the trivial solution $(0,0)$ is locally asymptotically stable for $\lambda<\lambda_{i}(i=1,2)$ 
and unstable for $\lambda>\lambda_{i}$, whereas both the solutions $\left(u^{*}(\mathbf{x}, \lambda), 0\right)$ and $\left(0, v^{*}(\mathbf{x}, \lambda)\right)$ are locally asymptotically stable where they exist (that is we have a supercritical bifurcation).

If the potential is such that $G_{u}, G_{v}$ have (first) strictly positive finite zeros, as it is in the case of the potential $G(u, v)$, then, by the maximum principle (Protter and Weiberger, 1984), both branches tend to a horizontal asymptote as $\lambda \rightarrow \infty$ in the bifurcation plane $(\lambda,|(u, v)|)$ (see Fig. 6.1).

For the potential $G(u, v)$ and for constant diffusion coefficients $D_{1}$ and $D_{2}$ the condition (5.7), when $v(\mathbf{x})$ is proportional to $u(\mathbf{x})$, takes the form

$$
k D_{2} u\left(1-u^{2}-a^{2} k^{2} u^{2}\right)=D_{1} k u\left(b^{2}-a^{2} u^{2}-c^{2} k^{2} u^{2}\right) .
$$

It is easy to see that the above equality holds if either

(i) $u(\mathbf{x}) \equiv 0$ (i.e. $k=\infty)$, or

(ii) $k=0$ (and hence $v(\mathbf{x}) \equiv 0$ ), or

(iii) $D_{2}\left(1-u^{2}-a^{2} k^{2} u^{2}\right)=D_{1}\left(b^{2}-a^{2} u^{2}-c^{2} k^{2} u^{2}\right)$.

Hence for the system (3.1) with the potential $G(u, v)$ and constant diffusion coefficients $D_{1}$ and $D_{2}$ a "proportionate" solution is possible in the following three cases:

(i) if $v(\mathbf{x}) \equiv 0$, then $k=0$. This is a case of a non-coexisting solution $\left(u^{*}(\mathbf{x}, \lambda), 0\right)$. The equation (6.1) for $u^{*}(\mathbf{x})$, together with boundary conditions, is to be solved.

(ii) if $u(\mathbf{x}) \equiv 0$, then $k=\infty$. This is also a case of a non-coexisting solution $\left(0, v^{*}(\mathbf{x}, \lambda)\right)$, and the equation (6.2) for $v^{*}(\mathbf{x})$, together with boundary conditions, is to be solved. 
(iii) since $k$ is constant and does not depend on $u(\mathbf{x})$, in this case a solution may exist only when $D_{1} b^{2}=D_{2}$, which gives $k^{2}=\left(D_{1} a^{2}-\right.$ $\left.D_{2}\right) /\left(D_{2} a^{2}-D_{1} c^{2}\right)$. In this case the equation

$$
D_{1} \nabla^{2} u=-\lambda u\left(1-u^{2}-a^{2} k^{2} u^{2}\right)
$$

for $u(\mathbf{x})$, together with boundary conditions, is to be solved, and $v(\mathbf{x})$ can be found from (5.5) thereafter. Of course, a non-trivial "proportionate" solution can only exist for $\lambda>\lambda_{c r}$, where $\lambda_{c r}$ is a first eigenvalue of the corresponding linear equation (Fife, 1979).

For the case of one spatial dimension and for $D_{1}=D_{2}=1$ all these three possibilities were considered by Norbury and Wake (1993), and the solutions were given there. More generally we refer here to a straightforward existence theory for this type of strongly elliptic boundary value problem (Amann, 1976).

\section{Bifurcations of NON-COEXisting SOlutions}

If $\left(u^{*}(\mathbf{x}, \lambda), v^{*}(\mathbf{x}, \lambda)\right)$ is a solution of the boundary value problem (3.1), (3.2), then, if $u=u^{*}+\xi$ and $v=v^{*}+\eta$, where the perturbations $\xi, \eta$ satisfy $|\xi|,|\eta| \ll 1$, we obtain the linearised equations for the perturbations

$$
\begin{aligned}
& \nabla\left(D_{1} \nabla \xi\right)+\mu\left(W_{u u}\left(u^{*}, v^{*}\right) \xi+W_{u v}\left(u^{*}, v^{*}\right) \eta\right)=0 \\
& \nabla\left(D_{2} \nabla \eta\right)+\mu\left(W_{v u}\left(u^{*}, v^{*}\right) \xi+W_{v v}\left(u^{*}, v^{*}\right) \eta\right)=0
\end{aligned}
$$

with homogeneous linear boundary conditions

$$
B_{1}(\xi)=0, \quad B_{22}(\eta)=0
$$


Here $\mu$ is an eigenvalue for the corresponding eigenvector $\left(\begin{array}{c}\xi \\ \eta\end{array}\right)$. Of course, the perturbed system is a gradient system as well. By (5.1), for the non-coexisting solution $(u(\mathbf{x}, \lambda), v(\mathbf{x}, \lambda))=\left(u^{*}(\mathbf{x}, \lambda), 0\right)$ mentioned in the previous section,

$$
W_{u v}\left(u^{*}, 0\right)=0
$$

and hence the equations for the perturbations $\xi, \eta$ uncouple:

$$
\begin{aligned}
& \nabla\left(D_{1} \nabla \xi\right)+\mu W_{u u}\left(u^{*}, 0\right) \xi=0 \\
& \nabla\left(D_{2} \nabla \eta\right)+\mu W_{v v}\left(u^{*}, 0\right) \eta=0 .
\end{aligned}
$$

For the potential $G(u, v), G_{u v}\left(u^{*}, 0\right)=0$ while $G_{u u}\left(u^{*}, 0\right)=\lambda\left(1-3 u^{*^{2}}\right)$ and $G_{v v}\left(u^{*}, 0\right)=\lambda\left(b^{2}-a^{2} u^{*^{2}}\right)$.

For there to be a nontrivial solution of the system $(7.1),(7.2)$, associated with a bifurcation of the non-coexisting solution of the system (3.1), (3.2), the constant $\mu$ has to be an eigenvalue of both equations (7.1) and (7.2) (Krasnosel'skii, 1964). This means that it is an extremely unlikely event: it is possible only on a set of measure zero in the system parameter space. The spectrum of each eigenvalue problem, (7.1) and (7.2), is a countable subset, $S_{i}$ where $i=1,2$ respectively, of real numbers, and hence is of measure zero. The spectrum of the system is the intersection of these two subsets, $S_{1} \cap S_{2}$, which could be empty. This intersection is non-empty, for example, when $D_{1}=D_{2}$ and $W_{u u}=W_{v v}$. For instance for the potential $G(u, v)$ the condition $W_{u u}=W_{v v}$ happens when $b=1$ and $a^{2}=3$.

However, when one of the two functions, either $\xi(\mathbf{x})$ or $\eta(\mathbf{x})$, is equal to zero, there is a countable set of eigenvalues $\mu_{j}^{1}$ where bifurcation is 
possible. That is there are bifurcations of $(\xi(\mathbf{x}), 0)$ and $(0, \eta(\mathbf{x}))$ types. A bifurcation of the first type, $(\xi(\mathbf{x}), 0)$, from the solution $\left(u^{*}(\mathbf{x}), 0\right)$ would imply a second similar non-coexisting solution. The second type, $(0, \eta(\mathbf{x}))$, leads to formation of a branch $\left(u^{*}(\mathbf{x}), \eta(\mathbf{x})\right)$ shown as a dashed line on the bifurcation diagram (see Fig. 7.1).

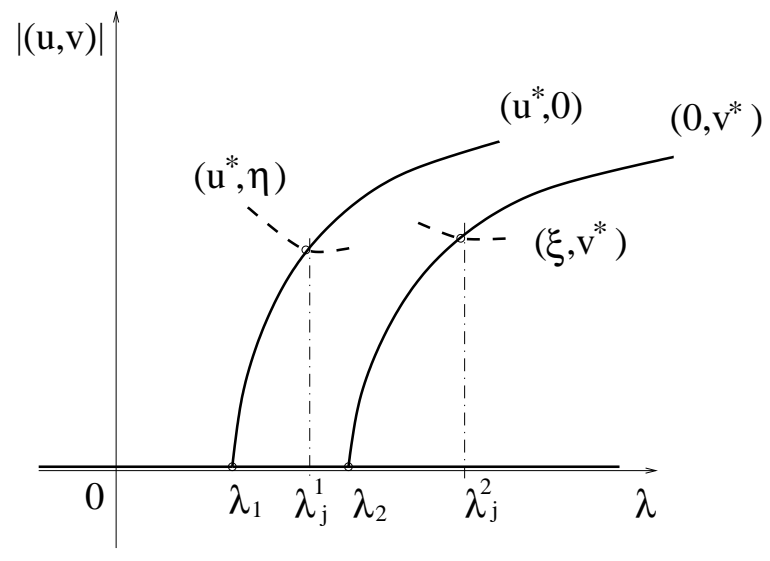

FiguRE 7.1. Bifurcation of non-coexisting solutions

Likewise for the non-coexisting solution $\left(0, v^{*}\right)$ there may be a bifurcation with a solution of the form $\left(\eta, v^{*}\right)$ (Fig. 7.1) at an eigenvalue $\lambda_{j}^{2}$ of the corresponding linearised equation

$$
\nabla\left(D_{1} \nabla \eta\right)+\lambda W_{u u}\left(0, v^{*}\right) \eta=0
$$

with the homogeneous boundary condition $B_{1}(\eta)=0$.

The global behaviour of these branches is unclear. They could join, or approach asymptotes as $\lambda$ tends to infinity (Rabinowitz, 1985, 1986). Also we cannot guarantee that for these branches both $u(\mathbf{x})$ and $v(\mathbf{x})$ remain non-negative. We suspect that changing of sign occurs.

\section{Conclusion}

With minor reservations, the results of the paper may be extended to unbounded regions, and the results are true for a system of $n$ variables. 
Thus we see that for quite general systems of this reaction-diffusion type arising from a double well potential $W(\mathbf{x}, u, v)$ the inclusion of reasonable spatial dependence in both the diffusion coefficients and in the reaction terms still does not permit a Hopf bifurcation or oscillatory solutions. However symmetrical bifurcations of stable non-coexisting (associated with the case when one species is absent while the other is present) branches generally occur, while secondary bifurcation to co-existing (possibly stable) states then becomes possible.

\section{REFERENCES}

Amann, H. (1976) Fixed point equations and nonlinear eigenvalue problems in ordered Banach spaces, SIAM Review, 18, 620-709.

Barbashin, E.A. (1970) Introduction to the theory of stability, WoltersNoordhoff, Groningen.

Fife, P.C. (1979) Mathematical aspects of reacting and diffusing systems, Springer, Berlin.

Krasnosel'ski, M.A. (1964) Topological methods in the theory of nonlinear integral equations, Pergamon, Oxford.

La Salle, J. and Lefschetz, S. (1961) Stability by Liapunov's direct method, Academic Press, New York.

Lyapunov, A.M. (1992) The General Problem of the Stability of Motion, Taylor \& Francis, London.

Murray, J.D. (1989) Mathematical Biology, Springer, Berlin (1989).

Norbury, J. and Wake, G.C. (1993) Spatial pattern formation for steady states of a population model, IMA J.Math.Appl.Med.Biol. 10, 19-30.

Okubo, A. et.al. (1989) On the spatial spread of the grey squirrel in Britain, Proc. R. Soc. Lond. B 238, 113-125. 
Protter, M.N. and Weinberger, H.F. (1984) Maximum principles in differential equations, Springer, New York.

Rabinowitz, P.H. (1985) Topological methods in bifurcation theory, University of Montreal, Montreal.

Rabinowitz, P.H. (1986) Minimax methods in critical point theory with applications to differential equations, Am. Math. Soc., Providence. 